\title{
Comparison of ionospheric radio occultation CHAMP data with IRI 2001
}

\author{
N. Jakowski and K. Tsybulya \\ Deutsches Zentrum für Luft-und Raumfahrt (DLR)/Institut für Kommunikation und Navigation, Kalkhorstweg 53, \\ D-17235 Neustrelitz, Germany
}

\begin{abstract}
GPS radio occultation measurements on board low Earth orbiting satellites can provide vertical electron density profiles of the ionosphere from satellite orbit heights down to the bottomside. Ionospheric radio occultation (IRO) measurements carried out onboard the German CHAMP satellite mission since 11 April 2001 were used to derive vertical electron density profiles (EDP's) on a routine basis. About 150 vertical electron density profiles may be retrieved per day thus providing a huge data basis for testing and developing ionospheric models. Although the validation of the EDP retrievals is not yet completed, the paper addresses a systematic comparison of about 78000 electron density profiles derived from CHAMP IRO data with the International Reference Ionosphere (IRI 2001).

The results are discussed for quite different geophysical conditions, e.g. as a function of latitude, local time and geomagnetic activity.

The comparison of IRO data with corresponding IRI data indicates that IRI generally overestimates the upper part of the ionosphere whereas it underestimates the lower part of the ionosphere under high solar activity conditions. In a first order correction this systematic deviation could be compensated by introducing a height dependence correction factor in IRI profiling.
\end{abstract}

\section{Introduction}

Low Earth Orbiting satellites carrying a dual frequency GPS receiver offer a unique chance to monitor the actual state of the global ionosphere on a continuous basis by GPS radio occultation measurements. No other profiling technique unifies profiling through the entire F2 layer with global coverage.

The German CHAMP satellite has been launched successfully on 15 July 2000 (Reigber et al., 2000) and has provided first ionospheric radio occultation measurements on 11 April
2001. Preliminary IRO retrieval and validation results are discussed by Jakowski et al. (2002). The vertical sounding based F2 layer height and electron density estimations agree within 13\% and 17\% RMS deviation, respectively.

The International Reference Ionospere (IRI) is a global empirical model of the most important parameters of the ionospheric plasma such as the electron density, electron and ion temperature, and ion composition. The IRI model is developed as a joint URSI/COSPAR project since 1972. A first IRI code was published in 1978 by Rawer et al. (1978). Over the years testing and modification of IRI has permanently continued resulting in essential improvements made available to the international research community through several versions (Bilitza et al., 1996). Considering the electron density, the data sources are mainly vertical sounding, incoherent scatter and in situ measurements onboard satellites (Bilitza, 2001). Ionospheric radio occultation measurements represent a new data type that should be helpful to improve IRI in the future.

In this paper we discuss a comparison of about 78000 electron density profiles derived from IRO measurements onboard CHAMP with corresponding model values computed from the International Reference Ionosphere.

\section{Radio occultation data basis}

The CHAMP science data are received in the DLR Remote Sensing Data Center Neustrelitz and than passed to the GeoForschungsZentrum (GFZ) Potsdam. A part of these data the IRO GPS data - are automatically processed in the Institute of Communications and Navigation of DLR by an operational data processing system (Wehrenpfennig et al., 2001). The computed higher-level data products are made available to the international science community via the Information and Science Data Center (ISDC) of GFZ Potsdam. 

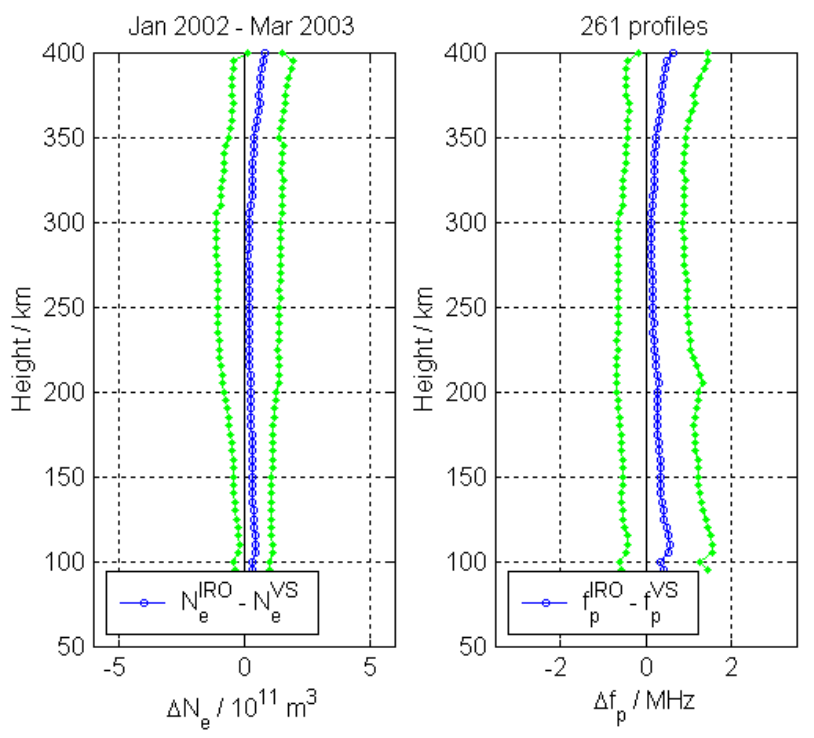

Fig. 1. Comparison of vertical electron density profiles derived from IRO data on CHAMP with vertical sounding data from the ionosonde station Juliusruh. Shown are the altitude dependent mean difference and the corresponding standard deviation. left: electron density, right: plasma frequency.

Due to the modular structure of the processing system high flexibility is achieved if retrieval modules shall be modified or replaced in the course of the CHAMP mission or if supplementary data shall be included.

To deduce vertical electron density profiles, a tomographic approach is established that uses spherically layered voxels with constant electron density (Jakowski, 1999; Jakowski et al., 2002).

Practically, the $1 \mathrm{~Hz}$ sampled dual frequency GPS measurements of TEC provide a system of equations for the electron density at the tangential points of the ray paths which is solved from above in a recursive way.

To overcome the difficulties arising due to the rather low orbit height of CHAMP $(<450 \mathrm{~km})$, the retrieval algorithm uses an adaptive model (Chapman layer superposed by a simple plasmasphere contribution) for estimating the topside ionosphere and plasmasphere above the CHAMP orbit.

From about 220 IRO measurements per day we retrieve about 150 vertical electron density profiles. For this comparative study with IRI 2001 we used about 78000 profiles, which have been provided by the automatic processing system so far.

Before starting this analysis we present some validation results obtained after comparing the retrieved electron density profiles with independent ionosonde data. Former comparisons of $\mathrm{fOF} 2$ and $\mathrm{hmF} 2$ data using the SPIDR data base have revealed bias values of $0.18 \mathrm{MHz}$ and $13.4 \mathrm{~km}$, respectively.

To evaluate the accuracy of the entire profile from the CHAMP orbit height down to the E-layer, the IRO data were compared with vertical sounding data from the vertical sounding stations Juliusruh $\left(53^{\circ} \mathrm{N} ; 13^{\circ} \mathrm{E}\right)$. The results are

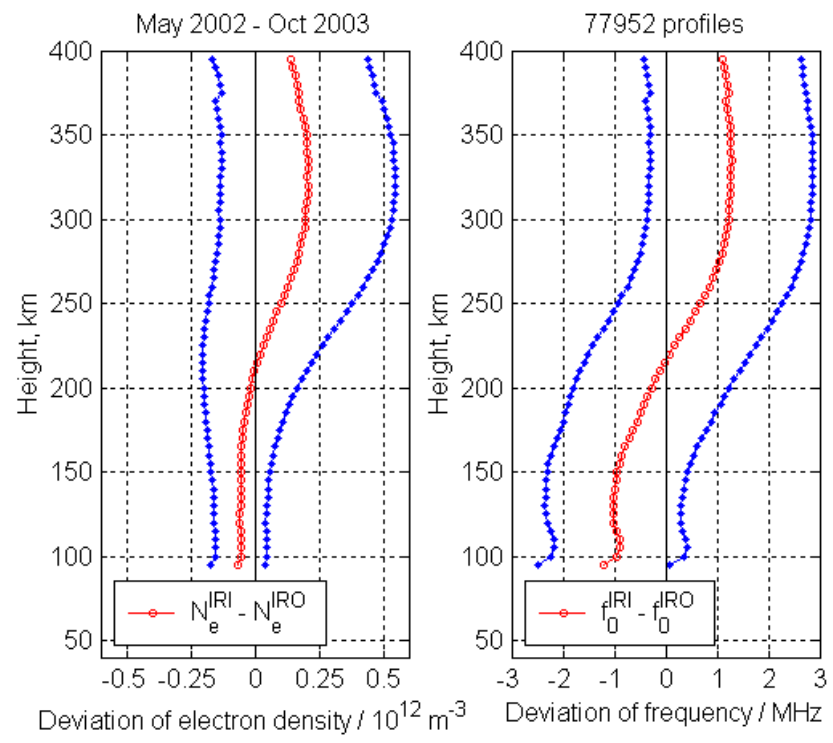

Fig. 2. Comparison of vertical electron density profiles derived from IRO data with corresponding profiles computed from IRI according to the difference EDP(IRI) - EDP(IRO) for all measurements obtained onboard CHAMP within the period May 2002October 2003.

shown in Fig. 1 and indicate a systematic positive bias of the IRO data of less than $0.8 \mathrm{MHz}\left(1 \times 10^{11} \mathrm{~m}^{-3}\right)$ and a standard deviation $\sigma$ from the mean of about $1 \mathrm{MHz}\left(1.3 \times 10^{11} \mathrm{~m}^{-3}\right)$ throughout the entire profile.

\section{The IRI model}

The IRI files were downloaded from NASA GSFC's National Space Science Center FTP server (ftp://nssdcftp.gsfc.nasa. gov/models/ionospheric/iri/iri2001/).

The source was translated with Fortran Power Station 4.0 compiler for PCs. To adapt the code to PC environment few insignificant changes in the code were made. None one of them changed the algorithms of the model.

To avoid low-level FORTRAN coding and facilitate generation of the graphs, MATLAB 6.0 was used for all high level programming. IRI 2001 was compiled to a dynamic link library, which had the main Ne calculating function exported and available for the MATLAB scripts. In all cases, ionospheric D-layer was not calculated (i.e. the faster and smaller IRI_SUB_NE function was used). In all cases, CCIR model was used for F peak, B0 option for the bottom side thickness and Standard option (no L condition) for estimating probability of F1 layer. Storm model was never used, as seriously impairing the processing speed. It's worth to notice that 12-month running mean sunspot number (Rz12) for year 2003, when the most of measurements were made, is based partially on measured $\mathrm{Rz}$ values for the past months and partially on predicted values for future months, so these predictions are implicitly included in the IRI calculations. 


\section{Results and discussion}

Keeping in mind the validation results of IRO derived electron density profiles for mid-latitudes, the comparison with IRI data should provide valuable information on the quality of the IRI model if deviations exceed the measured bias and the $1 \sigma$ dispersion range.

Figure 2 shows the bias and standard deviation between IRO and IRI data for more than one year of measurements from May 2002-October 2003. The comparison indicates a quite different behavior in the upper and lower part of the bottomside ionosphere. Whereas IRI generally exceeds the IRO measurements in the peak density range by about $1 \mathrm{MHz}\left(2 \times 10^{11} \mathrm{~m}^{-3}\right)$ IRI underestimates the IRO data by about $1 \mathrm{MHz}\left(5 \times 10^{11} \mathrm{~m}^{-3}\right)$ in the lower ionosphere $(100$ $150 \mathrm{~km})$. The standard deviation $\sigma$ is generally less than 1.5 MHz $\left(3.3 \times 10^{11} \mathrm{~m}^{-3}\right)$.

To study the latitudinal and local time dependence of the difference, the data set has been divided into three parts representing high $\left(|\phi|>60^{\circ}\right)$, medium $\left(30^{\circ}<|\phi|<60^{\circ}\right)$ and low latitude $\left(|\phi|<30^{\circ}\right)$ ranges. These data sets are subdivided in 4 groups corresponding to different local times (night: 21:00-03:00 LT, morning: 03:00-09:00 LT, day: 09:0015:00 LT, evening: 15:00-21:00 LT) as it is shown in Fig. 3. The mean deviation of the plasma frequency fp is generally less than $1.6 \mathrm{MHz}(\sigma=1.4 \mathrm{MHz})$ at high and less than $1.5 \mathrm{MHz}(\sigma=1.9 \mathrm{MHz})$ at mid- latitudes under day-time conditions above $300 \mathrm{~km}$ height. Surprisingly the corresponding bias reaches only $1.1 \mathrm{MHz}(\sigma=2.1 \mathrm{MHz})$ at low latitudes. It is difficult to comment this low latitude result because entire IRO derived electron density profiles have not yet been validated sufficiently for this latitude range. We are aware of the fact that the spherical symmetry assumption in IRO retrievals is violated in particular in the crest region due to strong horizontal gradients of the electron density distribution and therefore errors cannot be excluded. As expected, due to the enhanced ionization and variability at low latitudes in the evening hours, the bias and the dispersion reach high values in the order of 2 and $2.5 \mathrm{MHz}$, respectively. In the lower ionosphere (100-150 km height) bias and dispersion values are principally smaller than the corresponding $\mathrm{F} 2$ layer values. The smallest bias is found at low latitudes at day-time with $0.3 \mathrm{MHz}(\sigma=1.9 \mathrm{MHz})$ whereas the biggest value is found also at low latitudes in the evening hours with $1.9 \mathrm{MHz}(\sigma=1.9 \mathrm{MHz})$.

Considering the fact that the bias of the IRO measurements is slightly positive throughout the entire profile at least at mid-latitudes (Fig. 1), the IRI overestimation of the electron density in the F2 layer height range $\mathrm{km}$ is a clear finding at least at mid-latitudes. Due to the persistence of this feature at all latitude ranges and local times we assume that IRI generally overestimates the ionospheric ionization in the F2 layer height range under high solar activity conditions. Furthermore, it becomes evident that the IRI generally underestimates the electron density in the lower ionosphere in the $100-150 \mathrm{~km}$ height range.
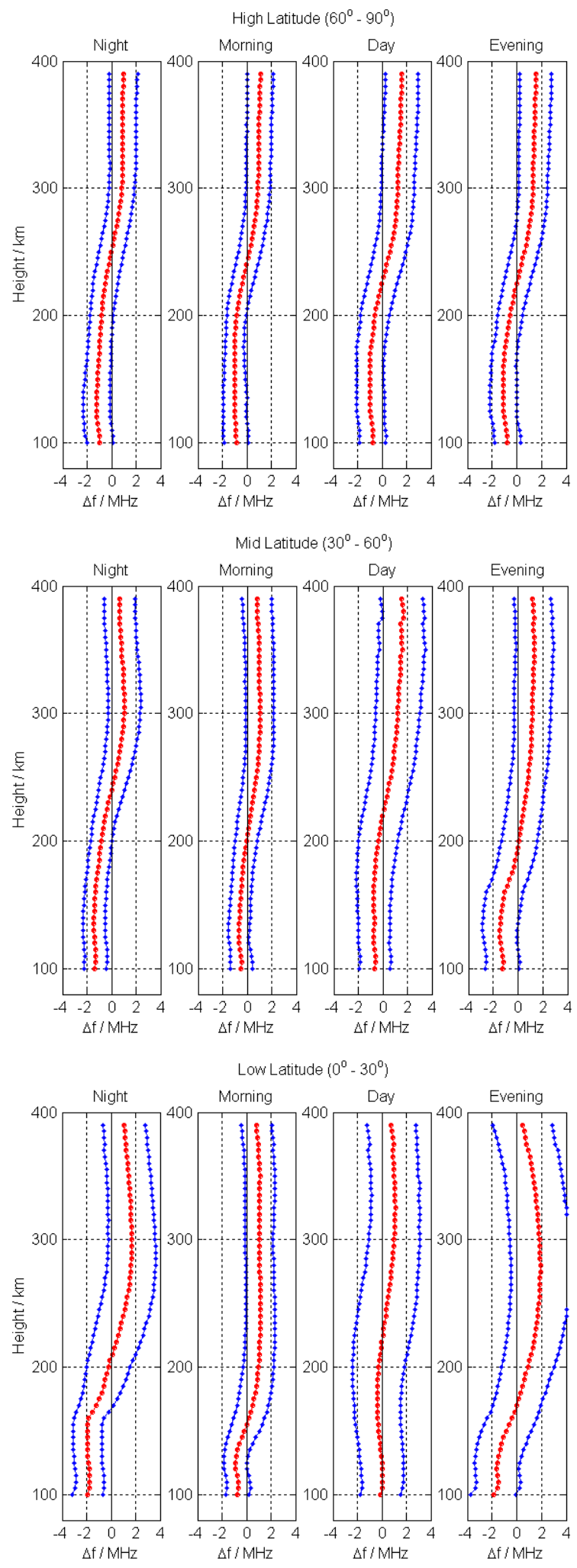

Fig. 3. Vertical profile of the difference $f p(I R I)-f p(I R O)$ for IRO measurements at high (a), middle (b) and low (c) latitude sectors obtained onboard CHAMP within the period April 2002-March 2003. 

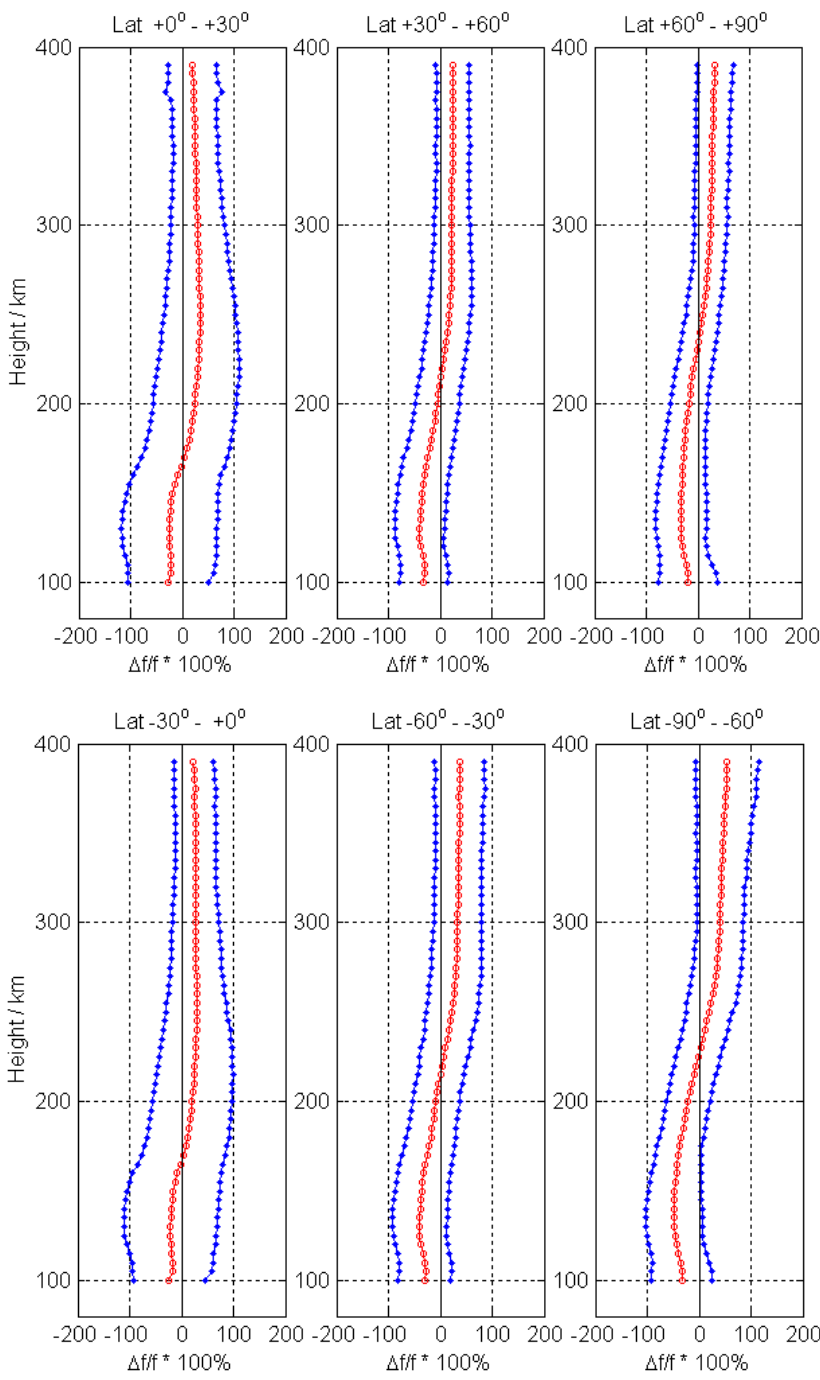

Fig. 4. Vertical profile of the percentage difference (fp(IRI) $\mathrm{fp}(\mathrm{IRO})) / \mathrm{fp}(\mathrm{IRO})$ for IRO measurements at different latitude sectors at northern (a) and southern (b) hemispheres obtained onboard CHAMP within the period May 2002-October 2003.

In order to check the consistency of the results, computations have been made separately for the northern and southern hemispheres. Figure 4 shows the latitudinal dependence of the percentage difference between plasma frequency data sets obtained at both hemispheres separately. Comparing bias and dispersion values of corresponding latitude ranges, no substantial difference can be found.

As expected, the percentage deviation is smallest at the F2 layer height range. At the northern hemisphere the bias and the standard deviation at $300 \mathrm{~km}$ height are generally less than $25 \%$ and $50 \%$, respectively.

The bias values are slightly enhanced in the southern hemisphere. At the lower ionosphere the bias is smallest with about $20 \%$ at both low latitude ionospheres accompanied by the highest dispersion value of about $90 \%$ found in the study.

It is important to mention that the main feature of the IRI - IRO difference (positive above $250 \mathrm{~km}$ height and negative

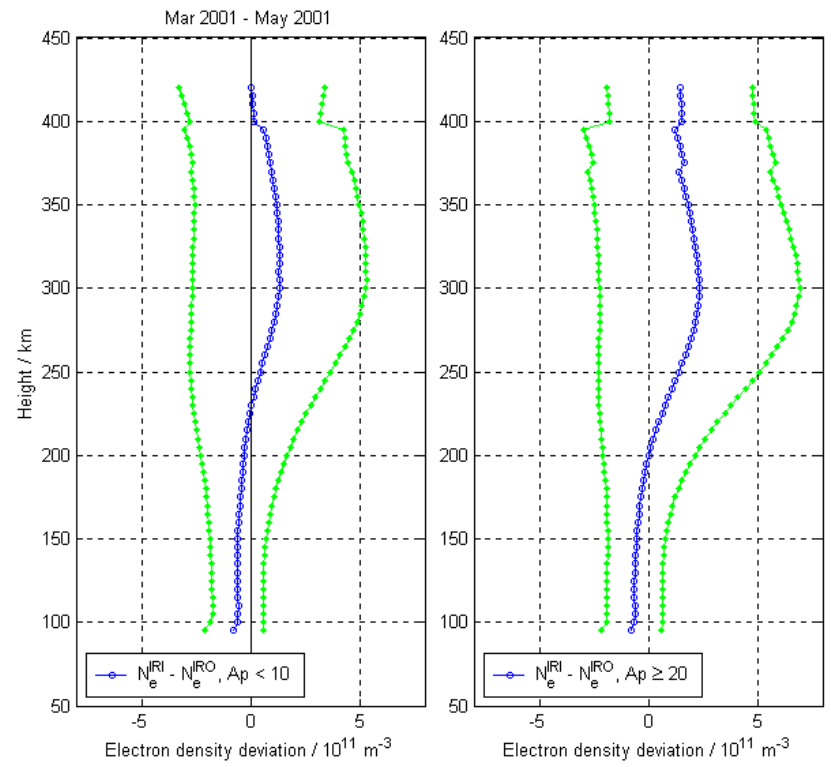

Fig. 5. Altitude profiles of the difference EDP(IRI) - EDP(IRO) between IRO derived electron densities and corresponding IRI values at different levels of geomagnetic activity $(\mathrm{Ap}<10$ and $\mathrm{Ap}>20)$ within the period March-May 2001.

at the lower ionosphere) is persistent at all latitude ranges independent from the hemisphere. The neutral line crossing height grows from about 170 at low latitudes via 210 at midto about $235 \mathrm{~km}$ at high latitudes.

As Fig. 5 shows, it is interesting to note that the bias between IRO retrievals and IRI model values and the corresponding dispersion significantly grow up under conditions of enhanced geomagnetic activity. This result is understandable because the described use of this IRI version doesn't include ionospheric perturbations. More detailed studies of this type could help to implement/improve perturbation terms in the IRI model.

\section{Summary and conclusions}

Electron density profiles retrieved from ionospheric GPS radio occultations measured onboard the CHAMP satellite since 11 April 2001 were compared with corresponding IRI model values. Preliminary retrieval results obtained by applying a model assisted retrieval technique are promising. Because the radio occultation technique presents a new data type, a systematic validation of data products must be continued.

The comparison of IRO data with corresponding IRI data indicates a systematic overestimation of the IRI derived electron density above $250 \mathrm{~km}$ height compared with the IRO data, whereas IRI underestimates the IRO retrievals of GPS measurements onboard CHAMP in the lower ionosphere at about $100-150 \mathrm{~km}$ height. Although the IRO measurements are not yet fully validated, and therefore the presented results have a preliminary character, we believe that the indicated 
general features of the IRI model compared with IRO retrievals might be helpful to improve IRI. So the height dependence of the IRI - IRO difference could simply be corrected by introducing an altitude dependent correction factor in IRI modeling.

Furthermore, the extraction of profile shape parameters such as $\mathrm{fOF} 2, \mathrm{hmF} 2$, and the bottomside slab thickness offer also unique chances for improving the IRI model.

It should be mentioned that the IRO derived vertical density profiles used in this study are directly taken from the automatically working IRO processing unit without further reviewing. A removal of unrealistic IRO outliers $(<0.5 \%)$ should principally lead to a better agreement between the IRI model data and the IRO measurements onboard CHAMP but would not essentially change the general finding.

Nevertheless, further improvements of the retrieval technique as e.g. the improvement of the topside ionosphere model are planned to enable more accurate observations.

Acknowledgements. The authors are very grateful to all the colleagues from the international CHAMP team keeping CHAMP in operation. We thank J. Mielich (IAP, Kühlungsborn) for providing vertical sounding data for comparison.

\section{References}

Bilitza, D.: International Reference Ionosphere 2000, Radio Sci., 36, 261-275, 2001.

Bilitza, D., Rawer K., Bossy L., and Gulyaeva T.: International Reference Ionosphere - Past, present and future, I, Electron density, Adv. Space Res., 13, 3, 23-32, 1996.

Jakowski, N.: Capabilities of radio occultation measurements onboard LEO satellites for ionospheric monitoring and research, Proc. 4th COST 251 Workshop "The Impact of the Upper Atmosphere on Terrestrial and Earth-Space Communications", edited by Vernon, A., 22-25 March, Funchal, Madeira, Portugal, 116121, 1999.

Jakowski, N., Wehrenpfennig A., Heise S., Reigber Ch., Lühr H., Grunwaldt L., and Meehan T. K.: GPS radio occultation measurements of the ionosphere from CHAMP: Early results, Geophysical Res. Lett., 29, 10, 95(1)-95(4), 2002.

Reigber, Ch., Lühr H., and Schwintzer P.: CHAMP mission status and perspectives, Suppl. to EOS, Transactions, AGU, 81, 48, F307, 2000.

Rawer, K., Ramakrishnan, S., and Bilitza, D.: International Reference Ionosphere 1978, URSI, Brussels, 1978.

Wehrenpfennig, A., Jakowski N., and Wickert J.: A Dynamically Configurable System for Operational Processing of Space Weather Data, Phys. Chem. Earth, 26, 601-604, 2001. 